Pacific

Journal of

Mathematics

ON RINGS WHICH ARE SUMS OF TWO PI-SUBRINGS: A COMBINATORIAL APPROACH

B. Felzenszwalb, A. Giambruno, and G. Leal 


\title{
ON RINGS WHICH ARE SUMS OF TWO PI-SUBRINGS: A COMBINATORIAL APPROACH
}

\author{
B. Felzenszwalb, A. Giambruno, and G. Leal
}

\begin{abstract}
We study the following open question: If a ring $R$ is the sum of two subrings $A$ and $B$ both satisfying a polynomial identity, does $R$ itself satisfy a polynomial identity? We give a positive answer to this question in case $R$ satisfies a special "mixed" identity or $(A B)^{k} \subseteq A$ for some $k \geq 1$ or $A$ or $B$ is a Lie ideal. Our approach is based on a comparative analysis of the sequences of codimensions of the three rings and their asymptotics. As a reward we obtain a bound on the degree of a polynomial identity satisfied by $R$ as a function of the degree of an identity satisfied by $A$ and $B$.
\end{abstract}

\section{Introduction.}

Let $R$ be a ring and suppose that $A$ and $B$ are two subrings of $R$ such that $R=A+B$ is their sum. Here we consider the following open question:

If both $A$ and $B$ satisfy a polynomial identity (i.e., PI-rings), does $R$ itself satisfy a polynomial identity?

The answer to this question is known to be positive in several cases. The first result that can be read in this setting is due to Kegel $[\mathbf{K}]$. He showed that if $A$ and $B$ are both nilpotent rings (so, they satisfy an identity of the type $\left.x_{1} x_{2} \ldots x_{n} \equiv 0\right)$ then the same conclusion holds in $R$. Bahturin and Giambruno in $[\mathbf{B G}]$ proved that if $A$ and $B$ are commutative rings then $R$ satisfies the identity $\left[x_{1}, x_{2}\right]\left[x_{3}, x_{4}\right] \equiv 0$, where $[x, y]=x y-y x$ is the Lie bracket. This result was later generalized by Beidar and Mikhalev in $[\mathbf{B M}]$. They proved that if both, $A$ and $B$ satisfy an identity of the form $\left[x_{1}, x_{2}\right] \ldots\left[x_{2 n-1}, x_{2 n}\right] \equiv 0$ for some $n \geq 2$, then $R$ is a PI-ring. By extending Kegel's result, Kepczyk and Puczylowski in [KP1] showed that if $A$ and $B$ are nil of bounded exponent (so, they satisfy an identity of the form $x^{n} \equiv 0$ ) then so is $R$. This result was later pushed further in [KP2] by proving that if one of the two subrings is nil of bounded exponent and the other is PI, then $R$ is PI.

To our knowledge these are the only results proved so far which hold without any further assumption on the structure of the $\operatorname{ring} R$ (or $A$ or $B$ ).

On the other hand, in [Ro1] Rowen proved that if $A$ and $B$ are both right (left) ideals of $R$, then an identity on both $A$ and $B$ forces $R$ to be a PI-ring. 
This result was later extended in [KP3] where the authors proved that the same conclusion holds if one requires that only $A$ or $B$ is a one-sided ideal. In case the ring $R$ is semiprime, the also showed that if $A$ is nil PI and $B$ is PI then $R$ is PI.

We remark that, except for some results about semiprime rings, only in $[\mathbf{K}]$ and $[\mathbf{B G}]$ an explicit identity of $R$ was exhibited. In all the other cases proved so far, the authors have shown the existence of an identity for $R$ without providing any information on its explicit form or on its degree (as a function of the degrees of an identity of $A$ and $B$ ). The reason for such failure is essentially the following: Most of these results use a reduction technique to the prime case where structure theory can be applied through the Martindale ring of quotients. Such reduction makes essentially use of the so called "Amitsur's trick" (see [Ro2]) which allows to pass from the semiprime case to the general case but gives no information on the degree of the identities so far found.

In this paper we answer this question in some special cases. Let $R=$ $A+B$, where $A$ and $B$ are subrings satisfying a polynomial identity.

We prove that if for some $k \geq 1$, either $(A B)^{k} \subseteq A$ or $(B A)^{k} \subseteq A$, then $R$ is PI. As a corollary we obtain the case when $A$ is a one-sided ideal or the case when $A B=B A$ and $B^{k} \subseteq A$. Moreover, $R$ is still PI if either $A$ or $B$ is a Lie ideal of $R$. We shall remark that this last result can also be derived from a theorem on special Lie algebras.

One can also consider, in a natural fashion, "mixed" identities or semiidentities for $R$, i.e., polynomials in two distinct sets of variables

$$
f\left(y_{1}, \ldots, y_{n}, z_{1}, \ldots, z_{m}\right)
$$

that vanish when we evaluate the $y_{i}$ 's into elements of $A$ and the $z_{i}$ 's into elements of $B$. We prove that $R$ is a PI-ring provided $R$ satisfies a $k$-special semi-identity i.e., an identity of the type $f\left(y_{1}, \ldots, y_{k}, z_{1}, \ldots, z_{k}\right)$, for some $k \geq 1$ where only one monomial of the type $y_{\sigma(1)} z_{\tau(1)} \ldots y_{\sigma(k)} z_{\tau(k)}$ appears with nonzero coefficient, for all $\sigma, \tau \in S_{k}$.

In all these results, we obtain an explicit function giving the degree of an identity for $R$ in terms of the degree of an identity of $A$ and $B$. Through this function, an explicit identity for $R$ can be constructed as it has been shown by Regev in [R2]. More precisely, suppose that $A$ and $B$ satisfy an identity of degree $d$ and one of the above hypotheses holds. Then we prove that $R$ satisfies an identity of degree $d^{\prime}$ where $d^{\prime}$ is the least integer greater than $a^{a}$ where $a$ has the following value: $a=8 e(d-1)^{4}$, if $A$ or $B$ is a Lie ideal; $a=8 e(k d(d-1)-1)^{2}(d-1)^{2}$, if $(A B)^{k} \subseteq A ; a=8 e(d-1)^{4}$, if $A$ is a one-sided ideal; $a=8 e k(d-1)^{2}$, if $R$ satisfies a $k$-special mixed identity of degree $k$ (here $e$ is the basis of the natural logarithms).

Our technique is based on a combinatorial approach to the problem using the sequence of codimensions of a ring. This sequence was introduced and exploited by Regev in $[\mathbf{R} 1]$. He proved, through this method, that the 
tensor product of two PI-rings is a PI-ring. In this paper we follow that approach and we attach to each of the rings $R, A$ and $B$ its codimension sequence and through the study of the relations among these sequences and their asymptotic behaviour we are able to prove our results. Unfortunately our approach does not solve the problem in its generality. We feel that one needs a better understanding through a deeper and throughout analysis of the sequences of codimensions and their relations.

\section{Preliminaries.}

Throughout we shall assume that all rings are algebras over a fixed field $F$. We make this assumption in order to simplify the notation. On the other hand it is easily verified that our results are still valid for general rings if one assumes that all polynomials have integer coefficients and that $A$ and $B$ satisfy an identity which is a monic polynomial. To this end we recall that by a theorem of Amitsur (see [Ro2]), if a ring satisfies an identity which is proper for all its homomorphic images, then it satisfies an identity of the type $\left(S t_{k}\right)^{l}$, for some $k, l$, where $S t_{k}$ is the standard polynomial of degree $k$.

Let $X=\left\{x_{1}, x_{2}, \ldots\right\}$ be a countable set and let $F\langle X\rangle$ be the free algebra on $X$ over $F$. Recall that a polynomial $f\left(x_{1}, \ldots, x_{n}\right)$ is an identity for the algebra $R$ if $f\left(r_{1}, \ldots, r_{n}\right)=0$ for all $r_{1}, \ldots, r_{n} \in R$ (in this case we write $f \equiv 0$ on $R$ ). In case $R$ satisfies a nontrivial identity $f$, i.e., $f \neq 0$, we say that $R$ is a PI-algebra.

In general one defines

$$
\operatorname{Id}(R)=\{f \in F\langle X\rangle \mid f \equiv 0 \text { on } R\},
$$

the set of polynomial identities of $R$. $\operatorname{Id}(R)$ has a structure of T-ideal of $F\langle X\rangle$ i.e., an ideal invariant under all endomorphisms of $F\langle X\rangle$; it is obvious that $R$ is a PI-algebra if and only if $\operatorname{Id}(R) \neq 0$.

Recall that a polynomial $f\left(x_{1}, \ldots, x_{n}\right)$ is multilinear if each variable $x_{i}$, $i=1, \ldots, n$, appears in every monomial of $f$ with degree one. Multilinear polynomials are important; in fact it is well-known that if $R$ satisfies an identity of degree $d$ then it satisfies a multilinear identity of degree $\leq d$.

For every $n \geq 1$ we define

$$
V_{n}=\operatorname{Span}_{F}\left\{x_{\sigma(1)} \ldots x_{\sigma(n)} \mid \sigma \in S_{n}\right\}
$$

where $S_{n}$ is the symmetric group of degree $n$. $V_{n}$ is the space of multilinear polynomials in $x_{1}, \ldots, x_{n}$. Since $\operatorname{dim}_{F} V_{n}=n$ !, from the above observation we easily get the following:

Remark 2.1. The algebra $R$ satisfies a polynomial identity if and only if there exists $n \geq 1$ such that

$$
\operatorname{dim}_{F} \frac{V_{n}}{V_{n} \cap \operatorname{Id}(R)}<n !
$$


The above remark, though trivial, will be essential not only in the proof of the existence of an identity for $R$ but also for the explicit computation of the corresponding degree.

\section{The basic reduction.}

From now on we shall assume that $R$ is an $F$-algebra such that $R=A+B$ for suitable subalgebras $A$ and $B$. We shall also assume that $A$ and $B$ are PI-algebras. For the sake of simplicity let us denote by $d$ the degree of an identity satisfied by $A$ and $B$.

Our first aim is to relate the valuations of polynomials in $A, B$ to those in $R$. To this end, we introduce two new countable sets $Y=\left\{y_{1}, y_{2}, \ldots\right\}$ and $Z=\left\{z_{1}, z_{2}, \ldots\right\}$. Then, we let $F\langle Y \cup Z\rangle$ be the free algebra on the set $Y \cup Z$ over $F$. We relate $F\langle Y \cup Z\rangle$ to $F\langle X\rangle$ by assuming that $x_{i}=y_{i}+z_{i}$, $i=1,2, \ldots$.

We can now define the notion of $s$-identity (or semi-identity) of $R$. A polynomial $f\left(y_{1}, \ldots, y_{n}, z_{1}, \ldots, z_{m}\right) \in F\langle Y \cup Z\rangle$ is an $s$-identity of $R$ if $f\left(a_{1}, \ldots, a_{n}, b_{1}, \ldots, b_{m}\right)=0$ for all $a_{1}, \ldots, a_{n} \in A, b_{1}, \ldots, b_{m} \in B$. Accordingly one defines

$$
\operatorname{Id}^{s}(R)=\{f \in F\langle Y \cup Z\rangle \mid f \text { is an } s \text {-identity of } R\},
$$

the ideal of $s$-identities of $R$. It is clear that $\operatorname{Id}^{s}(R)$ is an ideal invariant under all endomorphisms of $F\langle Y \cup Z\rangle$ that leave $F\langle Y\rangle$ and $F\langle Z\rangle$ invariant. Also $\operatorname{Id}(R), \operatorname{Id}(A), \operatorname{Id}(B) \subseteq \operatorname{Id}^{s}(R)$.

Now we need the notion of multilinear polynomial in $F\langle Y \cup Z\rangle$. To this end, we give the same degree one to the variables $y_{i}$ and $z_{i}$ for all $i=1,2, \ldots$. Then

$$
W_{n}=\operatorname{Span}_{F}\left\{w_{\sigma(1)} \ldots w_{\sigma(n)} \mid \sigma \in S_{n}, w_{i}=y_{i} \text { or } z_{i} \text {, for all } i=1, \ldots, n\right\}
$$

is the space of multilinear polynomials in $y_{1}, z_{1}, \ldots, y_{n}, z_{n}$. It is clear that $\operatorname{dim}_{F} W_{n}=2^{n} n$ ! and

$$
V_{n} \subseteq W_{n}
$$

Since

$$
\frac{V_{n}}{V_{n} \cap \operatorname{Id}(R)}=\frac{V_{n}}{V_{n} \cap\left(W_{n} \cap \operatorname{Id}^{s}(R)\right)} \cong \frac{V_{n}+\left(W_{n} \cap \operatorname{Id}^{s}(R)\right)}{W_{n} \cap \operatorname{Id}^{s}(R)} \subseteq \frac{W_{n}}{W_{n} \cap \operatorname{Id}^{s}(R)},
$$

we have the following:

Lemma 3.1. $\operatorname{dim}_{F} \frac{V_{n}}{V_{n} \cap \operatorname{Id}(R)} \leq \operatorname{dim}_{F} \frac{W_{n}}{W_{n} \cap \operatorname{Id}^{s}(R)}$.

At the light of Remark 2.1, we can now make the following reduction. Recall that $R$ satisfies an identity of degree $n$ if and only if $\operatorname{dim}_{F} \frac{V_{n}}{V_{n} \cap \operatorname{Id}(R)}<$ $n$ !

Remark 3.2. If there exists $n \geq 1$ such that $\operatorname{dim}_{F} \frac{W_{n}}{W_{n} \cap \operatorname{Id}^{s}(R)}<n$ !, then $R$ is a PI-algebra and satisfies an identity of degree $n$. 
The spaces $W_{n}$ are still too large for our computations. Hence we next make one further reduction.

Let $t \geq 0$ and fix integers $1 \leq r_{1} \leq \cdots \leq r_{t} \leq n$. Then define

$$
\begin{gathered}
V_{r_{1}, \ldots, r_{t}}=\operatorname{Span}_{F}\left\{w_{\sigma(1)} \ldots w_{\sigma(n)} \mid \sigma \in S_{n}, w_{i}=y_{i}, \text { for } i \in\left\{r_{1}, \ldots, r_{t}\right\},\right. \\
\left.w_{j}=z_{j}, \text { for } j \notin\left\{r_{1}, \ldots, r_{t}\right\}\right\} .
\end{gathered}
$$

Clearly

$$
W_{n}=\bigoplus_{1 \leq r_{1} \leq \cdots \leq r_{t} \leq n} V_{r_{1}, \ldots, r_{t}} .
$$

Also, it is easy to see that $W_{n} \cap \operatorname{Id}^{s}(R)=\bigoplus_{1 \leq r_{1} \leq \cdots \leq r_{t} \leq n}\left(V_{r_{1}, \ldots, r_{t}} \cap \operatorname{Id}^{s}(R)\right)$. It follows that

$$
\operatorname{dim}_{F} \frac{W_{n}}{W_{n} \cap \operatorname{Id}^{s}(R)}=\sum_{1 \leq r_{1} \leq \cdots \leq r_{t} \leq n} \operatorname{dim}_{F} \frac{V_{r_{1}, \ldots, r_{t}}}{V_{r_{1}, \ldots, r_{t}} \cap \operatorname{Id}^{s}(R)} .
$$

Write for simplicity $V_{1, \ldots, t}=V_{t, n-t}$ and notice that for all $1 \leq r_{1} \leq \cdots \leq$ $r_{t} \leq n, V_{r_{1}, \ldots, r_{t}} \cong V_{t, n-t}$ and $V_{r_{1}, \ldots, r_{t}} \cap \operatorname{Id}^{s}(R) \cong V_{t, n-t} \cap \operatorname{Id}^{s}(R)$. Since for every $t=0, \ldots, n$, there exist $\left(\begin{array}{c}n \\ t\end{array}\right)$ subspaces $V_{r_{1}, \ldots, r_{t}}$ isomorphic to $V_{t, n-t}$, we get:

Lemma 3.3. $\operatorname{dim}_{F} \frac{W_{n}}{W_{n} \cap \operatorname{Id}^{s}(R)}=\sum_{t=0}^{n}\left(\frac{n}{t}\right) \operatorname{dim}_{F} \frac{V_{t, n-t}}{V_{t, n-t} \cap \operatorname{Id}^{s}(R)}$.

We can now prove the final reduction.

Remark 3.4. In order to prove that $R$ is a PI-algebra, it is enough to prove that there exists $n \geq 1$ such that for all $t=0,1, \ldots, n$,

$$
\operatorname{dim}_{F} \frac{V_{t, n-t}}{V_{t, n-t} \cap \operatorname{Id}^{s}(R)}<\frac{n !}{2^{n}} .
$$

In this case $R$ satisfies an identity of degree $n$.

Proof. Suppose $\operatorname{dim}_{F} \frac{V_{t, n-t}}{V_{t, n-t} \cap \mathrm{Id}^{s}(R)}<\frac{n !}{2^{n}}$. Then, by the previous lemma,

$$
\operatorname{dim}_{F} \frac{W_{n}}{W_{n} \cap \operatorname{Id}^{s}(R)}<\sum_{t=0}^{n}\left(\begin{array}{l}
n \\
t
\end{array}\right) \frac{n !}{2^{n}}=2^{n} \frac{n !}{2^{n}}=n !
$$

and we are done by Remark 3.2.

\section{Ordering monomials.}

Let $1 \leq d \leq n$. Recall (see [R2]) that a permutation $\sigma \in S_{n}$ is called $d$-bad if there exist $1 \leq k_{1}<\cdots<k_{d} \leq n$ such that $\sigma\left(k_{1}\right)>\cdots>\sigma\left(k_{d}\right)$. We say that $\sigma$ is $d$-good if it is not $d$-bad. The $d$-good permutations are quite spare in $S_{n}$; in fact, as a consequence of a theorem of Dilworth one can prove the following: 
Lemma 4.1 ([R2, Theorem 1.8]). In $S_{n}$ the number of d-good permutations is $\leq \frac{(d-1)^{2 n}}{(d-1) !}$.

The $d$-good permutations were used in PI-theory for finding a bound to $\operatorname{dim}_{F} \frac{V_{n}}{V_{n} \cap \operatorname{Id}(C)}$ for a PI-algebra $C$.

We say that a monomial $x_{\sigma(1)} \ldots x_{\sigma(n)}$ is $d$-good if the corresponding permutation $\sigma$ is $d$-good. The result is the following:

Theorem 4.2 ([R2, Theorem 1.3]). Let $C$ be an algebra satisfying an identity of degree $d$. Then every monomial in $V_{n}$ can be written $(\bmod \cdot \operatorname{Id}(C))$ as a linear combination of $d$-good monomials. Hence $\operatorname{dim}_{F} \frac{V_{n}}{V_{n} \cap \operatorname{Id}(C)} \leq \frac{(d-1)^{2 n}}{(d-1) !}$.

Next step is to generalize the above theorem by adapting it to our situation $R=A+B$. Recall that we are assuming throughout that $A$ and $B$ satisfy an identity of degree $d$. In order to simplify the notation, we make the following:

Definition 4.3. Let $t \geq 0$ and write $w \in V_{t, n-t}$ in the following form:

$$
w=w_{1} y_{\sigma(1)} \ldots y_{\sigma\left(i_{1}\right)} w_{2} y_{\sigma\left(i_{1}+1\right)} \ldots y_{\sigma\left(i_{2}\right)} w_{3} \ldots w_{r} y_{\sigma\left(i_{r-1}+1\right)} \ldots y_{\sigma\left(i_{r}\right)} w_{r+1}
$$

where $w_{1}, \ldots, w_{r+1}$ are (eventually trivial) monomials in the variables $z_{i}$. If the permutation $\sigma$ is $d$-good (d-bad) we say that $w$ is $d$-y-good $(d$-y-bad resp.).

Recall that an additive subgroup $U$ of a ring $R$ is a Lie ideal of $R$ if for all $u \in U, r \in R$, we have that $[u, r] \in U$.

Lemma 4.4. Let $A$ be a Lie ideal of $R$. Then, for all $t=0,1, \ldots, n, V_{t, n-t}$ is spanned $\left(\bmod . \mathrm{Id}^{s}(R)\right)$ by all d-y-good monomials.

Proof. Suppose that the conclusion of the lemma is false. We first order the monomials of $V_{t, n-t}$ according to the left lexicographic order of the variables $y_{i}$. Then, among all monomials which do not satisfy the conclusion of the lemma, we pick a smallest one (in the given order). Let such monomial be

$$
w=w_{1} y_{\sigma(1)} \ldots y_{\sigma\left(i_{1}\right)} w_{2} \ldots w_{r} y_{\sigma\left(i_{r-1}+1\right)} \ldots y_{\sigma\left(i_{r}\right)} w_{r+1}
$$

In the monomial $w$ we first make the formal substitution

$$
y_{\sigma\left(i_{s}\right)} w_{s+1}=\bar{y}_{\sigma\left(i_{s}\right)}+w_{s+1} y_{\sigma\left(i_{s}\right)}
$$

for $s=1, \ldots, r$, where $\bar{y}_{\sigma\left(i_{s}\right)}=\left[y_{\sigma\left(i_{s}\right)}, w_{s+1}\right]$. Since $A$ ia a Lie ideal of $R$, the elements $\bar{y}_{\sigma\left(i_{s}\right)}$ evaluate to elements of $A$. It follows that we can write $w$ as a linear combination of monomials in the variables $y_{i}, \bar{y}_{i}, z_{i}$ where either some monomial $w_{i}$ has been absorbed in a $\bar{y}_{i}$ or it has been moved to the left past some $y_{i}$.

A repeated application of this process allows us to write $w$ as a linear combination of monomials of the type

$$
w^{\prime}=w_{1}^{\prime} y_{\sigma(1)}^{\prime} \ldots y_{\sigma\left(i_{1}\right)}^{\prime} y_{\sigma\left(i_{1}+1\right)}^{\prime} \ldots y_{\sigma\left(i_{2}\right)}^{\prime} \ldots y_{\sigma\left(i_{r}\right)}^{\prime}
$$


where $y_{j}^{\prime}=\left[y_{j}, w_{u_{1}}, \ldots, w_{u_{s}}\right]$ for some $u_{1}, \ldots, u_{s}, s \geq 0$ and $w_{1}^{\prime}$ is a monomial in the variables $z_{i}$. Note that in our terminology the Lie commutators are left normed i.e., $\left[x_{1}, \ldots, x_{n}\right]=\left[\left[x_{1}, x_{2}\right], \ldots, x_{n}\right]$. Also, in each monomial $w^{\prime}$, the permutation of the indices of the variables $y_{i}^{\prime}$ is still $\sigma$.

Since the conclusion of the lemma does not hold for the monomial $w$, then in particular $\sigma$ is a $d$-bad permutation. It follows that there exist $1 \leq j_{1}<\cdots<j_{d} \leq t$ such that $\sigma\left(j_{1}\right)>\cdots>\sigma\left(j_{d}\right)$. Write

$$
w^{\prime}=a y_{1}^{\prime \prime} y_{2}^{\prime \prime} \ldots y_{d}^{\prime \prime}
$$

where $a=w_{1}^{\prime} y_{\sigma(1)}^{\prime} \ldots y_{\sigma\left(j_{1}-1\right)}^{\prime}$ and

$$
y_{1}^{\prime \prime}=y_{\sigma\left(j_{1}\right)}^{\prime} \ldots y_{\sigma\left(j_{2}-1\right)}^{\prime}, \ldots, y_{d}^{\prime \prime}=y_{\sigma\left(j_{d}\right)}^{\prime} \ldots y_{\sigma\left(i_{r}\right)}^{\prime} .
$$

Let $f\left(y_{1}, \ldots, y_{d}\right)=\sum_{\tau \in S_{d}} \alpha_{\tau} y_{\tau(1)} \ldots y_{\tau(d)}$ be a multilinear identity of degree $d$ satisfied by $A$. We may clearly assume that $\alpha_{1}=1$. Since $A$ is a subring and a Lie ideal of $R$, then the polynomials $y_{1}^{\prime \prime}, \ldots, y_{d}^{\prime \prime}$ evaluate to elements of $A$. It follows that $f\left(y_{1}^{\prime \prime}, \ldots, y_{d}^{\prime \prime}\right)$ is an $s$-identity of $R$. Hence $f\left(y_{1}^{\prime \prime}, \ldots, y_{d}^{\prime \prime}\right) \in \operatorname{Id}^{s}(R) \cap V_{t, n-t}$.

Write

$$
w^{\prime}=a y_{1}^{\prime \prime} \ldots y_{d}^{\prime \prime} \equiv-\sum a \alpha_{\tau} y_{\tau(1)}^{\prime \prime} \ldots y_{\tau(d)}^{\prime \prime} \quad\left(\bmod \operatorname{Id}^{s}(R)\right) .
$$

By the definition of $y_{1}^{\prime \prime}, \ldots, y_{d}^{\prime \prime}$, since $\sigma$ is $d$-bad, it follows that each monomial $a y_{\tau(1)}^{\prime \prime} \ldots y_{\tau(d)}^{\prime \prime}$, to the right-hand side of $(1)$, is smaller that $a y_{1}^{\prime \prime} y_{2}^{\prime \prime} \ldots y_{d}^{\prime \prime}$ (in the left lexicographic order of the $y_{i}^{\prime \prime \prime}$ s).

If we now recall the definition of the $y_{i}$ 's and we open up all the brackets, we obtain that $w^{\prime}$ and, so, the original monomial $w$, can be written $\left(\bmod . \mathrm{Id}^{s}(R)\right)$ as a linear combination of monomials (in the variables $y_{i}$ and $z_{i}$ ) which are smaller than $w$ in the left lexicographic order of the $y_{i}$ 's. By the minimality of $w$, it follows that the lemma holds for such monomials. Hence each of them can be written as a linear combination $\left(\bmod . \operatorname{Id}^{s}(R)\right)$ of monomials which are $d$-y-good. But then the same conclusion holds for $w$ and this is a contradiction.

Lemma 4.5. Suppose that for some $k \geq 1,(A B)^{k} \subseteq A$. Then, for all $t=0,1, \ldots, n, V_{t, n-t}$ is spanned $\left(\bmod . \mathrm{Id}^{s}(R)\right)$ by all $k d(d-1)$-y-good monomials.

Proof. Suppose that the conclusion of the lemma is false and take, as before, a monomial $w$ which does not satisfy the conclusion of the lemma and is smallest in the order of the $y_{i}$ 's. Let

$$
w=w_{1} y_{\sigma(1)} \ldots y_{\sigma\left(i_{1}\right)} w_{2} \ldots w_{r} y_{\sigma\left(i_{r-1}+1\right)} \ldots y_{\sigma\left(i_{r}\right)} w_{r+1}
$$

By the choice of $w$, in particular, $\sigma$ is $k d(d-1)$-bad and let $1 \leq j_{1}<\cdots<$ $j_{k d(d-1)} \leq t$ be such that $\sigma\left(j_{1}\right)>\cdots>\sigma\left(j_{k d(d-1)}\right)$. Suppose first that we 
can write

$$
w=u y_{\sigma\left(j_{m}\right)} a_{1} y_{\sigma\left(j_{m+1}\right)} a_{2} \ldots a_{d} y_{\sigma\left(j_{m+d-1}\right)} v
$$

for some $m \in\{1,2, \ldots, k d(d-1)-d+1\}$, where $a_{1}, \ldots, a_{d}$ are monomials in the only variables $y_{i}$ and $u$ and $v$ are suitable monomials. Then

In this case set $y_{\sigma\left(j_{m}\right)} a_{1}=y_{1}^{\prime}, y_{\sigma\left(j_{m+1}\right)} a_{2}=y_{2}^{\prime}, \ldots, y_{\sigma\left(j_{m+d-1}\right)} v=y_{d}^{\prime}$.

$$
w=u y_{1}^{\prime} y_{2}^{\prime} \ldots y_{d}^{\prime}
$$

If $f\left(y_{1}, \ldots, y_{d}\right)$ is the multilinear identity of degree $d$ satisfied by $A$, then $f\left(y_{1}^{\prime}, \ldots, y_{d}^{\prime}\right)$ is still an identity of $A$ and, by applying it as in the previous lemma, we can write

$$
w \equiv-\sum_{\tau \in S_{d}} \alpha_{\tau} u y_{\tau(1)}^{\prime} \ldots y_{\tau(d)}^{\prime} \quad\left(\bmod . \operatorname{Id}^{s}(R)\right)
$$

for some $\alpha_{\tau} \in F$. But each monomial on the right-hand side of (2) is smaller than $w$ in the order of the variables $y_{i}$; hence we get, by the minimality of $w$, that each of them can be written $\left(\bmod \cdot \operatorname{Id}^{s}(R)\right)$ as a linear combination of $k d(d-1)$-y-good monomials. But then the same conclusion holds for $w$, a contradiction.

Hence we may assume that for any $d$ indices $j_{m}<j_{m+1}<\cdots<j_{m+d-1}$ (of the sequence giving the $k d(d-1)$-y-badness of $w$ ), in the monomial $w$, at least one variable $z_{i}$ appears between the variables $y_{\sigma\left(j_{m}\right)}$ and $y_{\sigma\left(j_{m+d-1}\right)}$.

Let us write $w$ in the form

$$
w=a_{0} y_{\sigma\left(j_{p_{1}}\right)} a_{1} y_{\sigma\left(j_{p_{2}}\right)} a_{2} \ldots y_{\sigma\left(j_{p_{d}}\right)} a_{d}
$$

where $p_{1}=1, a_{0}, a_{1}, \ldots, a_{d}$ are monomials in the variables $y_{i}$ and $z_{i}$ and $y_{\sigma\left(j_{p_{m}}\right)} a_{m}$ evaluates to either $(A B)^{k} A$ or $(A B)^{k}$, for all $m=1, \ldots, d$. In order for this decomposition to hold, we have to show that $p_{d} \leq k d(d-1)$. In fact, by the assumption made above, in $w$, at least one variable $z_{i}$ appears between two variables $y_{\sigma\left(j_{m}\right)}$ and $y_{\sigma\left(j_{m+d-1}\right)}$. Hence, for $i=1, \ldots, d-1$,

$$
p_{i+1}-p_{i} \leq k(d-1),
$$

but then, $p_{d}=1+\left(p_{2}-p_{1}\right)+\cdots+\left(p_{d}-p_{d-1}\right) \leq k d(d-1)$ as claimed. Write now

$$
w=a_{0} y_{1}^{\prime} y_{2}^{\prime} \ldots y_{d}^{\prime}
$$

where $y_{1}^{\prime}=y_{\sigma\left(j_{p_{1}}\right)} a_{1}, \ldots, y_{d}^{\prime}=y_{\sigma\left(j_{p_{d}}\right)} a_{d}$ and let $f\left(y_{1}, \ldots, y_{d}\right)$ be the identity of $A$. Recall that $y_{i}^{\prime}=y_{\sigma\left(j_{p_{i}}\right)} a_{i}$ evaluates to $(A B)^{k} A$ or $(A B)^{k}$ and both these sets lie in $A$ by hypothesis. Hence $f\left(y_{1}^{\prime}, \ldots, y_{d}^{\prime}\right)$ is an $s$-identity of $R$ and $f\left(y_{1}^{\prime}, \ldots, y_{d}^{\prime}\right) \in \operatorname{Id}^{s}(R) \cap V_{t, n-t}$.

Through the identity $f\left(y_{1}^{\prime}, \ldots, y_{d}^{\prime}\right)$ we can now rearrange $\left(\bmod . \operatorname{Id}^{s}(R)\right)$ the variables $y_{i}^{\prime}$ in $w$ and, as above, this leads to a contradiction due to the minimality of $w$. 
Corollary 4.6. If $A$ is a one-sided ideal of $R$ then, for all $t=0,1, \ldots, n$, $V_{t, n-t}$ is spanned $\left(\bmod . \mathrm{Id}^{s}(R)\right)$ by all d-y-good monomials.

Proof. From the previous lemma we get that $V_{t, n-t}$ is spanned by the $d(d-$ 1 )-y-good monomials. We next show that this result can be improved as claimed in the conclusion of the corollary. Suppose $A$ is a one-sided ideal of $R$.

As in the previous lemma we take $w$ smallest in the order of the $y_{i}$ 's for which the conclusion does not hold. We take $1 \leq j_{1}<\cdots<j_{d} \leq t$ such that $\sigma\left(j_{1}\right)>\cdots>\sigma\left(j_{d}\right)$.

Then we write

$$
w=a y_{1}^{\prime} y_{2}^{\prime} \ldots y_{d}^{\prime}
$$

where $a=w_{1} y_{\sigma(1)} \ldots y_{\sigma\left(j_{1}-1\right)} w_{1}^{\prime}$ and

$$
y_{1}^{\prime}=y_{\sigma\left(j_{1}\right)} \ldots y_{\sigma\left(j_{2}-1\right)} w_{2}^{\prime}, \ldots, y_{d}^{\prime}=w_{1} y_{\sigma\left(j_{d}\right)} \ldots w_{r+1}
$$

with $w_{1}^{\prime}, w_{2}^{\prime}, \ldots$ eventually trivial monomials in the variables $z_{i}$. Since $A$ is a right ideal of $R$, the monomials $y_{1}^{\prime}, \ldots, y_{d}^{\prime}$ evaluate to elements of $A$. Hence they can be rearranged $\left(\bmod . \mathrm{Id}^{s}(R)\right)$ using the identity of $A$. This completes the proof as in the previous lemmas.

\section{Mixed identities.}

In this section we examine the case when $R$ further satisfies a semi-identity $f \in \operatorname{Id}^{s}(R)$ of a special type. It is clear, by the standard multilinearization process, that if $R$ satisfies a nontrivial semi-identity of degree $m$, then it also satisfies a multilinear one of degree $\leq m$. If $\left\{i_{1}, \ldots, i_{k}\right\}$ is a subset of $\{1, \ldots, n\}$ we denote by $S_{k}\left(i_{1}, \ldots, i_{k}\right)$ the subgroup of $S_{n}$ of all permutations fixing $\{1, \ldots, n\} \backslash\left\{i_{1}, \ldots, i_{k}\right\}$. Also, in order to simplify the notation, we write the variables $z_{t+1}, \ldots, z_{n}$ of $V_{t, n-t}$ as $z_{1}, \ldots, z_{n-t}$, respectively. We now make the formal definition.

Definition 5.1. Let $f\left(y_{1}, \ldots, y_{k}, z_{1}, \ldots, z_{k}\right) \in F\langle Y \cup Z\rangle$ be a multilinear polynomial. We say that $f$ is $k$-special if

$$
f\left(y_{1}, \ldots, y_{k}, z_{1}, \ldots, z_{k}\right)=y_{1} z_{1} \ldots y_{k} z_{k}+\sum_{\substack{\sigma \in S_{2 k} \\ \sigma \notin T}} \alpha_{\sigma} w_{\sigma(1)} \ldots w_{\sigma(2 k)}
$$

for some $\alpha_{\sigma} \in F$, where, for $i=1, \ldots k, w_{2 i}=z_{i}, w_{2 i-1}=y_{i}$ and $T=$ $S_{k}(1,3, \ldots, 2 k-1) \times S_{k}(2,4, \ldots, 2 k) \subseteq S_{2 k}$.

In few words, $f$ is $k$-special if the only monomial of the type

$$
y_{\sigma(1)} z_{\tau(1)} \ldots y_{\sigma(k)} z_{\tau(k)}
$$

appearing in $f$ with nonzero coefficient is $y_{1} z_{1} \ldots y_{k} z_{k}$.

We next prove that in the presence of a $k$-special semi-identity for $R$ we can bound exponentially the dimension of $V_{t, n-t}\left(\bmod . \mathrm{Id}^{s}(R)\right)$. Recall that 
if $n \geq 1$ is an integer such that $n=r_{1}+\cdots+r_{p}, r_{i}>0$, then the multinomial coefficient is defined as $\left(\begin{array}{c}n \\ r_{1}, \ldots, r_{p}\end{array}\right)=\frac{n !}{r_{1} ! \ldots r_{p} !}$.

Lemma 5.2. Suppose that $R$ satisfies a $k$-special semi-identity. Then for all $t=0,1, \ldots, n$,

$$
\operatorname{dim}_{F} \frac{V_{t, n-t}}{V_{t, n-t} \cap \operatorname{Id}^{s}(R)} \leq \frac{\left(4 k(d-1)^{2}\right)^{n}}{(d-1) !} .
$$

Proof. We decompose the space $V_{t, n-t}$ as follows: Write $t=q_{1}+\cdots+$ $q_{p}, n-t=r_{1}+\cdots+r_{p}$ where $q_{1} \geq 0, r_{p} \geq 0$, for $p>1$ we have that $q_{2}, \ldots, q_{p}, r_{1}, \ldots, r_{p-1}$ are positive integers and at most one between $q_{1}$ and $r_{p}$ can eventually be zero. Then define $(\mathbf{q}, \mathbf{r})=\left(q_{1}, \ldots, q_{p}, r_{1}, \ldots, r_{p}\right)$ and

$$
\begin{aligned}
& U_{(\mathbf{q}, \mathbf{r})}^{(p)}=\operatorname{Span}_{\mathrm{F}}\left\{y_{\sigma(1)} \ldots y_{\sigma\left(q_{1}\right)} z_{\tau(1)} \ldots z_{\tau\left(r_{1}\right)} \ldots y_{\sigma\left(q_{1}+\cdots+q_{p-1}+1\right)}\right. \\
&\left.\ldots y_{\sigma(t)} z_{\tau\left(r_{1}+\cdots+r_{p-1}+1\right)} \ldots z_{\tau(n-t)} \mid \sigma \in S_{t}, \tau \in S_{n-t}\right\} .
\end{aligned}
$$

We write

$$
\bigoplus_{(\mathbf{q}, \mathbf{r})} U_{(\mathbf{q}, \mathbf{r})}^{(p)}=U^{(p)}
$$

Clearly $V_{t, n-t}=\oplus_{p \geq 1} U^{(p)}$.

We claim that for every $(\mathbf{q}, \mathbf{r})$ and for every $p$,

$$
U_{(\mathbf{q}, \mathbf{r})}^{(p)} \subseteq \bigoplus_{s \leq 2 k} U^{(s)}
$$

In fact, suppose the above inclusion is false and pick a subspace $U_{(\mathbf{q}, \mathbf{r})}^{(p)}$ with $p$ minimal such that $U_{(\mathbf{q}, \mathbf{r})}^{(p)}$ is not contained in the right-hand side of (3). Since $p>2 k$, every monomial in $U_{(\mathbf{q}, \mathbf{r})}^{(p)}$ is of the form

$$
v=v_{1} u_{1} \ldots v_{p} u_{p}
$$

where $v_{1}, \ldots, v_{p}$ are monomials in the variables $y_{i}, u_{1}, \ldots, u_{p}$ are monomials in the variables $z_{i}$ and either $v_{1}$ or $u_{p}$ is eventually trivial. Suppose for short that $v_{1} \neq 1$. Since, by hypothesis, $R$ satisfies a $k$-special semi-identity and $A$ and $B$ are subrings, we get that

$$
v_{1} u_{1} \ldots v_{k} u_{k} \equiv \sum_{\substack{\sigma \in S_{2 k} \\ \sigma \notin T}} \alpha_{\sigma} w_{\sigma(1)} \ldots w_{\sigma(2 k)} \quad\left(\bmod . \operatorname{Id}^{s}(R)\right),
$$

where $w_{2 i}=v_{i}$ and $w_{2 i-1}=u_{i}$, for $i=1, \ldots, k$. But then, this says that $\left(\bmod . \mathrm{Id}^{s}(R)\right) v$ is a linear combination of monomials belonging to some $U_{\left(\mathbf{s}_{1}, \mathbf{r}_{1}\right)}^{\left(p_{1}\right)}$ with $p_{1}<p$. By the minimality of $p, U_{\left(\mathbf{s}_{1}, \mathbf{r}_{\mathbf{1}}\right)}^{\left(p_{1}\right)} \subseteq \bigoplus_{q \leq 2 k} U^{(p)}$ and, since $U_{(\mathbf{s}, \mathbf{r})}^{(p)} \subseteq U_{\left(\mathbf{s}_{1}, \mathbf{r}_{\mathbf{1}}\right)}^{\left(p_{1}\right)}$, we get a contradiction. 
We now compute $\operatorname{dim}_{F} U_{(\mathbf{q}, \mathbf{r})}^{(p)}$ for every $p \leq 2 k$. Since both $A$ and $B$ satisfy an identity of degree $d$, by Theorem 4.2 , any monomial in the only variables $y_{i}$ (in the only variables $z_{i}$ ) can be written $\left(\bmod . \operatorname{Id}^{s}(R)\right)$ as a linear combination of $d$-good monomials. Moreover, by Lemma 4.1, the number of $d$-good monomials in $m$ variables is bounded by $\frac{(d-1)^{2 m}}{(d-1) !}$. Since in every monomial of $U_{(\mathbf{q}, \mathbf{r})}^{(p)}$ occur $q_{1}$ consecutive variables $y_{i}, \ldots, q_{p}$ consecutive variables $y_{i}\left(r_{1}\right.$ consecutive variables $z_{i}, \ldots, r_{p}$ consecutive variables $\left.z_{i}\right)$, we get that $U_{(\mathbf{q}, \mathbf{r})}^{(p)}$ is spanned $\left(\bmod . \operatorname{Id}^{s}(R)\right)$ by at most

$$
\begin{aligned}
& \frac{(d-1)^{2 q_{1}}}{(d-1) !} \ldots \frac{(d-1)^{2 q_{p}}}{(d-1) !} \frac{t !}{q_{1} ! \ldots q_{p} !} \frac{(d-1)^{2 r_{1}}}{(d-1) !} \ldots \frac{(d-1)^{2 r_{p}}}{(d-1) !} \frac{(n-t) !}{r_{1} ! \ldots r_{p} !} \\
& \leq \frac{(d-1)^{2 n}}{((d-1) !)^{2 p-1}}\left(\begin{array}{c}
t \\
q_{1}, \ldots, q_{p}
\end{array}\right)\left(\begin{array}{c}
n-t \\
r_{1}, \ldots, r_{p}
\end{array}\right)
\end{aligned}
$$

monomials.

Recall that if $m, m_{1}, \ldots, m_{p}$ are positive integers such that $m=m_{1}+\cdots+$ $m_{p}$, by the multinomial theorem (see $[\mathbf{B i}]$ ), we have that $\left(\begin{array}{c}m \\ m_{1}, \ldots, m_{p}\end{array}\right) \leq$ $p^{m}$. Hence, since $p \leq 2 k$, we get that $U_{(\mathbf{q}, \mathbf{r})}^{(p)}$ is spanned $\left(\bmod \cdot \operatorname{Id}^{s}(R)\right)$ by at most

$$
\frac{(d-1)^{2 n}}{(d-1) !}(2 k)^{t}(2 k)^{n-t}=\frac{(d-1)^{2 n}(2 k)^{n}}{(d-1) !}
$$

monomials. Since there are at most $2^{n}$ spaces $U_{(\mathbf{q}, \mathbf{r})}^{(p)}$ inside $V_{t, n-t}$, the conclusion follows.

\section{The main results.}

In the next lemma we prove that the results obtained in Lemma 4.4, Lemma 4.5 and Corollary 4.6 allow us to get a suitable upper bound to the dimension of $V_{t, n-t}$ (modulo $V_{t, n-t} \cap \operatorname{Id}^{s}(R)$ ).

Lemma 6.1. Let $0 \leq t \leq n$ and suppose that $V_{t, n-t}$ is spanned (mod. $\left.\operatorname{Id}^{s}(R)\right)$ by the $c$-y-good monomials, for some $c \geq 1$. Then,

$$
\operatorname{dim}_{F} \frac{V_{t, n-t}}{V_{t, n-t} \cap \operatorname{Id}^{S}(R)} \leq 2^{n}(c-1)^{2 t}(d-1)^{2(n-t)}(t+1)^{n-t} .
$$

Proof. For $t=q_{1}+\cdots+q_{p}$ and $n-t=r_{1}+\cdots+r_{p}$, let $U_{(\mathbf{s}, \mathbf{r})}^{(p)}$ be the subspace of $V_{t, n-t}$ defined in the proof of the Lemma 5.2.

We shall compute the dimension of the spaces $U_{(\mathbf{q}, \mathbf{r})}^{(p)}$ and, so, the dimension of $V_{t, n-t}\left(\bmod . \operatorname{Id}^{s}(R)\right)$, for $t=0,1, \ldots$ By hypothesis $U_{(\mathbf{q}, \mathbf{r})}^{(p)}$ is generated $\left(\bmod . \mathrm{Id}^{s}(R)\right)$ by all $c$-y-good monomials. By Lemma 4.1 the number of 
such monomials is bounded by $\frac{(c-1)^{2 t}}{(c-1) !}$. On the other hand, by recalling that $B$ satisfies an identity of degree $d$, by Theorem 4.2, every monomial in the only variables $z_{i}$ can be written $\left(\bmod . \mathrm{Id}^{s}(R)\right)$ as a linear combination of $d$-good monomials. Since in every monomial of $U_{(\mathbf{q}, \mathbf{r})}^{(p)}$ occur $r_{1}$ consecutive $z_{i}, \ldots, r_{p}$ consecutive $z_{i}$, it follows that $U_{(\mathbf{s}, \mathbf{r})}^{(p)}$ is spanned $\left(\bmod . \operatorname{Id}^{s}(R)\right)$ by at most

$$
\begin{aligned}
& \frac{(c-1)^{2 t}}{(c-1) !} \frac{(n-t) !}{r_{1} ! \ldots r_{p} !} \frac{(d-1)^{2 r_{1}}}{(d-1) !} \ldots \frac{(d-1)^{2 r_{p}}}{(d-1) !} \\
& \leq \frac{(c-1)^{2 t}(d-1)^{2(n-t)}}{((c-1) !(d-1) !)^{p-1}}\left(\begin{array}{c}
n-t \\
r_{1}, \ldots, r_{p}
\end{array}\right) \\
& \leq(c-1)^{2 t}(d-1)^{2(n-t)} p^{n-t}
\end{aligned}
$$

monomials (note that the last inequality holds since by the multinomial theorem $\left.\left(\begin{array}{c}n-t \\ r_{1}, \ldots, r_{p}\end{array}\right) \leq p^{n-t}\right)$.

Recalling that $p \leq t+1$ and that there exist at most $2^{n}$ subspaces $U_{(\mathbf{q}, \mathbf{r})}^{(p)}$, we get the desired conclusion.

Theorem 6.2. Let $R=A+B$ be a ring which is the sum of two subrings $A$ and $B$ and suppose that $A$ and $B$ both satisfy an identity of degree $d$. We have:

1) If $(A B)^{k} \subseteq A$, for some $k \geq 1$, then $R$ is PI and satisfies an identity whose degree is the least integer greater than $a^{a}$ where $a=8 e(k d(d-$ $1)-1)^{2}(d-1)^{2}$; in case $A$ is a one-sided ideal then we can take $a=$ $8 e(d-1)^{4}$;

2) if $A$ is a Lie ideal, then $R$ is PI and satisfies an identity whose degree is the least integer greater than $a^{a}$ where $a=8 e(d-1)^{4}$;

$3)$ if $R$ satisfies a $k$-special semi-identity, for some $k \geq 1$, then $R$ is PI and satisfies an identity whose degree is the least integer greater than $8 e k(d-1)^{2}$.

Proof. By Remark 3.4 it is enough to prove that there exists $n$ such that

$$
\operatorname{dim}_{F} \frac{V_{t, n-t}}{V_{t, n-t} \cap \operatorname{Id}^{S}(R)}<\frac{n !}{2^{n}},
$$

for all $t=0, \ldots, n$. The smallest $n$ for which this inequality holds will also give us the desired degree of an identity for $R$.

In order to get a bound for this smallest $n$, we are going to use the wellknown inequality (see, for instance, [FR, p. 105]) that holds for any $x \geq 1$ :

$$
\left(\frac{x}{e}\right)^{x}<\frac{\Gamma(x+1)}{\sqrt{2 \pi x}}<\Gamma(x+1)
$$


where $\Gamma(x+1)$ is the gamma function (recall that $\Gamma(n+1)=n$ ! for every natural number $n$ ) and $e$ is the basis of the natural logarithms.

Suppose that $V_{t, n-t}$ is spanned $\left(\bmod . \operatorname{Id}^{s}(R)\right)$ by the $c$ - $y$-good monomials, for some $c \geq 1$. Then, according to Lemma 6.1, it is enough to find a natural number $n$ such that

$$
2^{n}(c-1)^{2 t}(d-1)^{2(n-t)}(t+1)^{n-t}<\frac{n !}{2^{n}}
$$

or, in view of the above remark,

$$
(4 e)^{n}(c-1)^{2 t}(d-1)^{2(n-t)}(t+1)^{n-t} \leq n^{n} .
$$

If we define $a=8 e(c-1)^{2}(d-1)^{2}$ then, since $t+1 \leq 2 t$ and $t \leq n$, all we need is $n$ such that

$$
a^{n} t^{n-t} \leq n^{n} .
$$

Here we have two possibilities: If $t \leq n / a$ clearly the inequality holds for every natural number $n \geq a$. If $n \geq t>n / a$ it is easy to check that the above inequality still holds for every natural number $n \geq a^{a}$.

By Lemma 4.4, in case $A$ is a Lie ideal of $R, V_{t, n-t}$ is spanned $\left(\bmod \cdot \operatorname{Id}^{s}(R)\right)$ by the $d$-y-good monomials. Hence by what we have just proved, by taking $c=d$, it follows that in this case $R$ is PI and it satisfies an identity of degree the least integer greater than $a^{a}$ where $a=8 e(d-1)^{4}$. Similarly, in case $(A B)^{k} \subseteq A$, by invoking Lemma 4.5 and $c=k d(d-1)$ above, we get an identity for $R$ of degree the least integer greater than $a^{a}$ where $a=8 e(k d(d-1)-1)^{2}(d-1)^{2}$.

Suppose now that $R$ satisfies a $k$-special semi-identity. Then, by Lemma 5.2 , we know that

$$
\operatorname{dim}_{F} \frac{V_{t, n-t}}{V_{t, n-t} \cap \operatorname{Id}^{s}(R)} \leq \frac{\left(4 k(d-1)^{2}\right)^{n}}{(d-1) !} .
$$

Therefore it is enough to take $n \geq 8 e k(d-1)^{2}>\frac{8 e k(d-1)^{2}}{\sqrt[n]{(d-1) !}}$.

One last remark is in order. As the referee has pointed out, the result: If $A$ and $B$ are PI and one of them is a Lie ideal then $R$ is PI, can also be proved directly by an application of the theory of special Lie algebras (see [B, Section 6.3]). In order to see this, regard $R$ as a Lie algebra under the bracket operation [, ] and $A$ and $B$ as Lie subalgebras. Since a Lie subalgebra of an associative PI-algebra is Lie PI ([B, Section 6.3]), both $A$ and $B$ satisfy a nontrivial identity as Lie algebras. If, say, $A$ is a Lie ideal, then $R / A$ is isomorphic to $B$ which is Lie PI. Then the Lie algebra $R$, being an extension of a PI-ideal $A$ by a PI-quotient algebra $B$ is PI. This clearly implies that $R$ is $\mathrm{PI}$ as an associative algebra. 


\section{References}

[B] Y. Bahturin, Identical Relations in Lie Algebras, VNU Science Press, Utrecht, 1987, MR 88f:17032, Zbl 0691.17001.

[BG] Y. Bahturin and A. Giambruno, Identities of sums of commutative subalgebras, Rend. Cir. Mat. Palermo (2), 43 (1994), 250-258, MR 96g:16032, Zbl 0829.16014.

[BM] K.I. Beidar and A.V. Mikhalev, Generalised polynomial identities and rings which are sums of two subrings, Algebra i Logica, 34 (1995), 3-11 (Russian); English translation: Algebra and Logica, 34 (1995), 1-5, MR 96i:16038, Zbl 0846.16016.

[Bi] N.L. Biggs, Discrete Mathematics, Clarendon Press, Oxford, 1989, MR 91h:00002, Zbl 0682.05001.

[FR] O.J. Farrell and B. Ross, Solved Problems: Gamma and Beta Functions, Legendre Polinomials, Bessel Functions, Dover Publications, New York, 1971, Zbl 0285.33003.

[K] O.H. Kegel, Zur nilpotenz gewisser assoziativer ringe, Math. Ann., 149 (1962/63), 258-260, MR 28 \#3049, Zbl 0106.25402.

[KP1] M. Kepczyk and E.R. Puczylowski, On radicals of rings which are sums of two subrings, Arch. Math., 66 (1996), 8-12, MR 96k:16033, Zbl 0860.16016.

[KP2] _ Rings which are sums of two subrings, J. Pure Applied Algebra, 133 (1998), 151-162, MR 2000a:16050, Zbl 0929.16017.

[KP3] _ Rings which are sums of two subrings satisfying a polynomial identity, Comm. Algebra, 29 (2001), 2059-2065, MR 2002g:16038, Zbl 0993.16017.

[R1] A. Regev, Existence of identities in $A \otimes B$, Israel J. Math., 11 (1972), 131-152, MR 47 \#3442, Zbl 0249.16007.

[R2] - The representations of $S_{n}$ and explicit identities of PI-algebras, J. Algebra, 51 (1978), 25-40, MR 57 \#9745, Zbl 0374.16009.

[Ro1] L.H. Rowen, Generalized polynomial identities II, J. Algebra, 38 (1976), 380-392, MR 57 \#3189a, Zbl 0324.16016.

[Ro2] - Polynomial Identities in Ring Theory, Academic Press, New York, 1980, MR 82a:16021, Zbl 0461.16001.

Received October 15, 2001 and revised April 16, 2002. The second author was partially supported by Murst of Italy and CNPq of Brazil; the third author was partially supported by CNPq of Brazil.

Instituto DE MATEMÁtica

Universidade Federal do Rio de JANEIRO

RiO DE JANEIRO

BRAZIL

E-mail address: bfel@terra.com.br

Dipartimento di Matematica ed Applicazioni

Università Di PALERMO

Via ARChIRAFI 34

90123 PALERMo

ITALY 
E-mail address: a.giambruno@unipa.it

Instituto De Matemática

UNIVERSIDADE FEDERAL DO RIO DE JANEIRO

RIO DE JANEIRO

BRAZIL

E-mail address: gleal@acd.ufrj.br 